\title{
Les kadoi à poix du Bruttium
}

\section{Laëtitia Cavassa}

\section{Résumé}

Cet article se propose de reprendre la question des conteneurs ayant transporté la poix du Bruttium. Jusqu'à présent, ces conteneurs étaient uniquement connus par la découverte de timbres mis au jour en Calabre et à Pompéi et mentionnant la poix du Bruttium (PIX BRUT), mais la forme complète nous faisait défaut. En comparant ces données avec les découvertes faites en Italie du Sud (au large de Tarente), nous nous proposons d'identifier les conteneurs ayant transporté la poix aux kadoi mentionnés dans les tablettes en bronze de Locres. Ces kadoi correspondraient à des conteneurs piriformes, à large ouverture, sans anse et pourvus d'un petit fond en bouton. Leurs production et circulation sont pour l'instant attestées entre le ler siècle avant J.-C. et le ler siècle de notre ère.

\section{Citer ce document / Cite this document :}

Cavassa Laëtitia. Les kadoi à poix du Bruttium. In: Mélanges de l'École française de Rome. Antiquité, tome $120, \mathrm{n}^{\circ} 1$. 2008. Antiquité. pp. 99-107;

doi : https://doi.org/10.3406/mefr.2008.10417

https://www.persee.fr/doc/mefr_0223-5102_2008_num_120_1_10417

Fichier pdf généré le 03/09/2018 


\title{
Les kadoi à poix du Bruttium
}

\author{
Laëtitia CAVAssA*
}

En souvenir de Marie-Pierre Amarger

Les sources littéraires sur la poix, un des produits les plus courants de l'économie antique, sont relativement abondantes. Elles nous renseignent sur les emplois de ce goudron végétal, sur les procédés de fabrication et sur les régions productrices, la Gaule', la Grèce ${ }^{2}$ et surtout le Bruttium. Rares, en revanche, sont les indications qu'on peut en tirer sur son commerce mais, sur ce point, l'archéologie commence à apporter des données nouvelles. Tel est l'objectif de notre présente contribution ${ }^{3}$.

La poix est un goudron végétal obtenu par traitement thermique des bois résineux. Les Grecs la nomment $\pi i \sigma \sigma \alpha$ les Latins pix. Un bref rappel des usages les plus fréquents s'impose dès l'abord. La poix est avant tout un goudron qui, liquéfié par chauffage, rend étanche aussi bien les vases en céramique que les coques de bois. Elle sert donc à l'imperméabilisation des amphores et tous récipients de terre cuite nécessaires à la production ${ }^{4}$, au transport et à la conservation du vin ${ }^{5}$. L'application d'une couche de poix parfait l'étanchéité des conteneurs en bois, notamment des tonneaux, et des coques de navire. Outre ces usages courants, la

*. Université de Provence-IRAA/Centre Jean Bérard. Nous remercions X. Lafon, J.-P. Brun, M.-B. Carre, P. Nunzi et R. Auriemma, pour leurs conseils et précisions fournies.

1. Athenée, Deipnosophistes, II, 206.

2. Théophraste, Recherches sur les plantes, IX, II, 3 et IX, 4, 1.

3. Les données textuelles et économiques concernant la poix, bien connues, ne seront que brièvement mentionnées. Notre contribution se veut novatrice quant à l'apport de données nouvelles concernant les conteneurs à poix du Bruttium.

4. Pline l'Ancien, Histoire naturelle, Livre XIV, 57, 127, 134.

5. Columelle, De l'agriculture, Livre XII, 18, 5-6.

6. Énée le Tacticien, Poliorcétique, XXXIII et XXXV.

7. Calpurnius Siculus, Bucoliques, 78-85.

8. Pline l'Ancien, XIV, 123 et Juvénal, Satires, IX, 15-18.

9. Lucrèce, De la nature, III, 1015-1020.

10. Ch. Feyel, Les artisans dans les sanctuaires grecs aux époques classique et hellénistique à travers la documentation financière en Grèce, BEFAR 318, Athènes, 2006. Quarante-neuf inscriptions relatives aux comptes financiers des sanctuaires d'Eleusis, d'Epidaure et de Délos mentionnent l'achat régu- poix était utilisée comme arme de guerre ${ }^{6}$, pour le marquage du bétail ${ }^{7}$, pour l'épilation masculine ${ }^{8}$, pour la torture ${ }^{9}$, comme pigment, pour l'entretien des édifices religieux ${ }^{10}$.

\section{LES PROCÉDÉS DE FABRICATION}

Deux techniques de préparation de la poix sont connues. La première est le gemmage qui consiste à recueillir la résine de pin et à la chauffer ${ }^{11}$. Pline l'Ancien la définit de la manière suivante : "On ouvre le picea du côté du soleil, non par une incision, mais par une entaille ôtant l'écorce sur deux pieds au plus, à une coudée au moins du sol. On n'épargne pas le bois lui-même, comme on fait pour les autres 'résineux', puisque les éclats servent. Mais on estime les plus voisins du sol; les éclats plus élevés donnent de l'amertume. Tout le liquide afflue alors de l'arbre entier dans la plaie...» ${ }^{12}$. La seconde méthode est la distillation des branches de résineux : «en Europe, la poix liquide s'obtient de la taeda par le feu; elle sert entre autres nombreux usages, à enduire les navires. Son lier de poix. Parmi celles-ci, quatre provenant d'Eleusis (ÉL 3 p. 59, ÉL 83 p. 71, ÉL 106, p. 75, ÉL 134, p. 78), trois d’Épidaure (ÉPI 31 p. 123, ÉPI 82 p. 133 et ÉPI 311 p. 174), et dixsept de Délos (DÉL 4 p. 189, DÉL 24 p. 193, DÉL 38 p. 195, DÉL 157 p. 219, DÉL 208 p. 227, DÉL 242 p. 235, DÉL 269 p. 240, DÉL 310 p. 247, DÉL 325 p. 250, DÉL 332 p. 251, DÉL 347 p. 253 , DÉL 429 p. 268 , DÉL 435 p. 268 , DÉL 438 p. 269, DÉL 526 p. 282, DÉL 535 p. 284 et DÉL 720 p. 316) mentionnent son utilisation pour vernir les boiseries et tuiles des édifices sacrés.

11. Une récente étude de P. Vivacqua, fait état de l'utilisation dans le Bruttium, de plats en céramique commune produits sur l'île de Pantelleria (la Pantellerian ware), particulièrement adaptés aux chocs thermiques pour chauffer ou réchauffer la poix. Voir P. Vivacqua, La produzione della pece nel Bruttium: nuovi dati alla luce della ceramica da fuoco, dans Annali della facoltà di lettere e filosofia, Università di Siena, vol. XXV, Firenze, 2004, p. 1-25.

12. Pline l'Ancien, Histoire naturelle, Livre XVI, 57, traduction J. André, Paris, 1962, (Collection des Universités de France). 
bois fendu en menus morceaux bouillonne dans les fours complètement entourés de feux extérieurs. Un premier distillat coule comme de l'eau par un canal $»^{13}$. Il s'agit de la distillation per descensum dont le principe consiste en la consumation de la matière à distiller (ici le bois de résineux) dont on tire un distillat liquide (la poix). Les principaux exemples archéologiquement connus de fours à poix antiques se trouvent en Gaule. Ceux fouillés dans les Causses par E. Loir ont permis une meilleure compréhension des techniques de production ${ }^{14}$. Ses découvertes sont une parfaite illustration de la technique présentée par Pline l'Ancien : une jarre enterrée est recouverte par une seconde mise têtebêche. Cette dernière contient les bûchettes de bois de résineux, calées par une claie en bois, et elle est percée d'un orifice pour permettre l'évacuation de la fumée. Le joint entre les deux jarres est luté par des boudins d'argile. Un feu est ensuite allumé autour de la jarre supérieure. La chaleur entraîne l'exsudation du distillat. La résine issue du bois se transforme alors en poix. À la fin de l'opération, la poix est récupérée au fond de la jarre inférieure (fig. 1). D'autres exemples montrent que le bas de la jarre enterrée est parfois percé d'un orifice relié à un canal permettant à la poix de s'écouler vers un bassin de récupération.

La poix obtenue pouvait être utilisée sur place ou expédiée vers les lieux de consommation. Les fouilles effectuées sur plusieurs épaves montrent qu'elle était commercialisée soit sous forme de pains ou de blocs ${ }^{15}$, soit sous forme liquide, dans des amphores de récupération ${ }^{16}$. Jusqu'à présent, les données disponibles n'indiquaient pas de type d'amphore ou de conteneur spécifiquement dédié au transport de la poix. Dans les épaves répertoriées par A.-J. Parker, les amphores de transport sont toutes de remploi. Par exemple, dans l'épave de Torre la Sal en Espagne, datée des années 130-110 avant notre ère, la cargaison comptait

13. Pline l'Ancien, XVI, 52.

14. E. Loir, L'industrie de la résine dans les Causses à l'époque galloromaine, Nancy, 1940. Voir également A. Trintignac, La production de poix dans la cité des Gabales (Lozère) à l'époque Galloromaine, dans $R A P, 2003$, p. 239-248.

15. Plusieurs épaves dont la cargaison était composée de pains ou de blocs de poix sont répertoriées par A. J. Parker (Ancient shipwreacks of the Mediterranean and the Roman provinces, Oxford, 1992 (BAR International Series, 580); citons l'épave de la Chrétienne 1 au large des côtes varoises, datée du I ${ }^{\text {er }}$ siècle de notre ère, ou l'épave du Dramont $\mathrm{C}$ datée de la fin du $\mathrm{II}^{\mathrm{e}}$ av. J.-C.

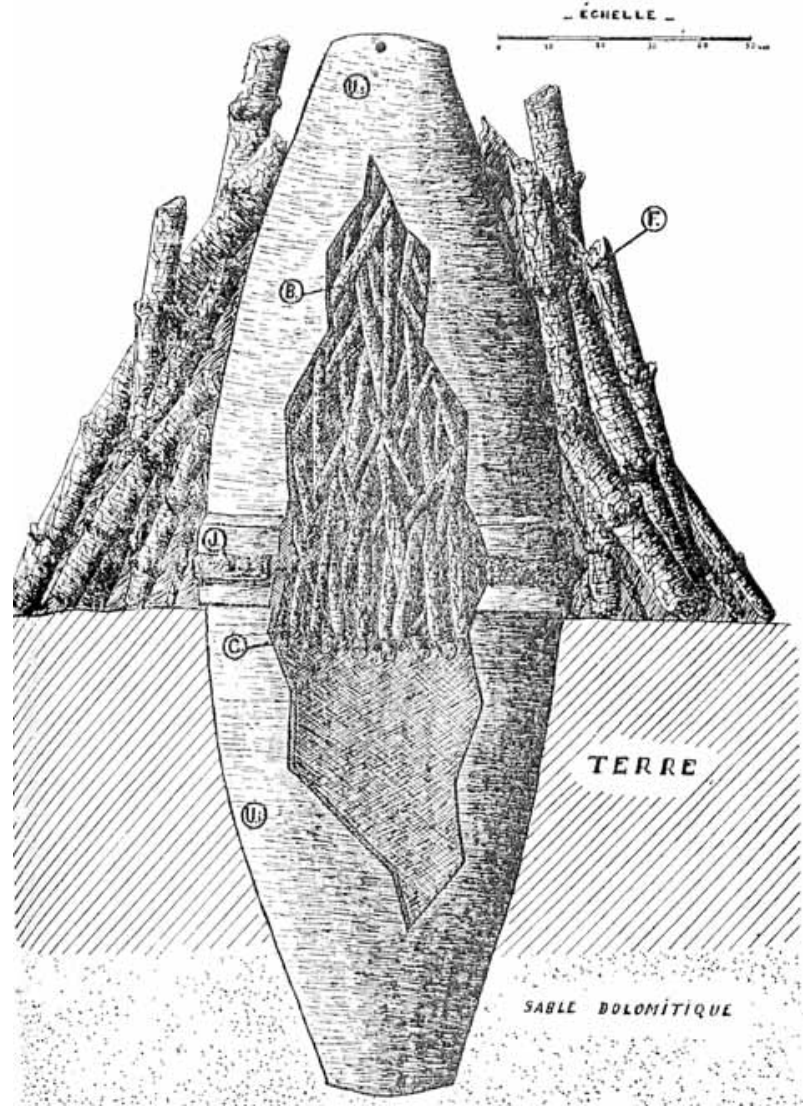

Fig. 1 - Coupe transversale d'un four à poix dans les Causses (d'après E. Loir 1940, p. 99, fig. 16).

vingt amphores italiques de type Dressel 1A remplies de poix et scellées par un bouchon en plâtre et fibres végétales ${ }^{17}$.

\section{LA POIX DU BRUTTIUM}

\section{Les textes}

La préparation et l'usage de la pix bruttia sont mentionnés par les sources à partir du $\mathrm{II}^{\mathrm{e}}$ siècle avant notre ère et jusqu'au $\mathrm{VI}^{\mathrm{e}}$ siècle de notre ère.

16. Par exemple l'épave de Nord-Levant au large des côtes varoises, contenait un chargement d'amphores gréco-italiques remplies au trois quart de poix semi-liquide. Voir J.-P. Joncheray, L. Long, L'épave profonde Héliopolis 2-Nord Levant (Var, -80m). Une fouille d'épave à l'aide de plongeurs à saturation et d'un sous-marin d'observation, dans Cahiers d'archéologie subaquatique 14, 2002, p. 131-160. De même, L. Long mentionne une épave au large de Marseille (Sud Caveaux 1) chargée d'amphores italiques de type Lamboglia 2 remployées pour transporter de la poix. Voir L. Long dans H.-G. Delauze et alii, dans Bulletin du DRASSM 2005, p. 97.

17. Parker, op. cit., p. $428, \mathrm{n}^{\circ} 1160$. 
Certains auteurs la citent sans donner de précisions, à l'instar de Columelle ${ }^{18}$, tandis que d'autres sont plus prolixes. L'indication la plus ancienne concerne Caton l'Ancien. Plutarque rapporte que le célèbre censeur aurait acheté des "entreprises de production de poix [...] desquelles il tirait de grands profits ${ }^{19}$.

Un passage de Cicéron est particulièrement instructif quant à l'organisation de cette exploitation.

Je me rappelle, à Smyrne, avoir recueilli de la bouche de Publius Rutilius Rufus les propos que voici : dans les débuts de sa jeunesse, il arriva qu'en vertu d'un sénatus-consulte, les consuls (Publius Scipion et Decimus Brutus, je crois) eurent à poursuivre un crime affreux et gigantesque. Un massacre avait été commis dans le massif forestier de la Sila, des hommes connus avaient été tués, et des soupçons pesaient sur une troupe d'esclaves, pour une part aussi des ingénus, relevant d'une société qui tenait des censeurs Publius Cornelius et Lucius Mummius l'exploitation des fonderies de poix. Le sénat donc avait décidé que les consuls auraient à instruire l'affaire et à trancher. Ce fut, dans le sens des publicains, une plaidoirie soignée, comme il ne cessait pas d'en avoir l'habitude, distinguée, que prononça Laelius. ${ }^{20}$

Denys d'Halicarnasse, relatant un fait du $\mathrm{III}^{\mathrm{e}}$ siècle avant notre ère, signale la poix du Bruttium comme "la plus odorante et la plus onctueuse»" précise : «les Bruttiens, après leur soumission volontaire aux Romains ${ }^{22}$, leur remirent la moitié de la zone montagneuse qu'on appelle Sila [...] De l'affermage de cette forêt le Peuple Romain tire chaque année d'importants revenus». Calpurnius Siculus, au I ${ }^{\text {er }}$ siècle de notre ère, évoque la dure

18. Columelle, De l'agriculture, Livre XII, 18, 5 et 22, 2.

19. Plutarque, Vies parallèles, Caton l'ancien, XXI, 5, traduction R. Flacelière et E. Chambry, Paris, 1969 (Collection des Universités de France).

20. Cicéron, Brutus, XXII, 85-86, traduction J. Martha, Paris, 1966 (Collection des Universités de France).

21. Denys d'Halicarnasse, Rome et la conquête de l'Italie aux IVe et $I I I^{e}$ siècle avant J.-C., traduction S. Pittia, Paris, 2002 (Collection Fragments Les Belles Lettres), p. 396-398.

22. La soumission du Bruttium à Rome est généralement datée de 272 avant J.-C.

23. Calpurnius Siculus, Bucoliques, 78-85, éd. J. Amat, Paris, 1991, (Collection des Universités de France).

24. Pline l'Ancien, op. cit, Livre XIV, 127.

25. S. Accardo, Villae romanae nell'Ager Bruttius. Il paesaggio rurale calabrese durante il dominio romano, Rome, 2000, p. 45. poix du Bruttium ${ }^{23}$. Pline l'Ancien déclare que «la poix d'Italie la plus estimée pour les récipients vinaires est celle du Bruttium» ${ }^{24}$.

Les témoignages ne se cantonnent pas aux $\mathrm{II}^{\mathrm{e}}$ et $\mathrm{I}^{\mathrm{er}}$ siècles avant et après J.-C. Encore au V ${ }^{\mathrm{e}}$ siècle de notre ère, Vibo Valentia était la principale localité d'expédition de la poix et du bois pour Rome ${ }^{25}$. Au cours de la deuxième moitié du $\mathrm{V}^{\mathrm{e}}$ et du début du $\mathrm{VI}^{\mathrm{e}}$ siècle, Cassiodore vante les productions de la Calabre : huile, fromages de la Sila, viande, bois et poix ${ }^{26}$. D'ailleurs, au XVII ${ }^{e}$ siècle, la poix, toujours fabriquée en Calabre, constituait l'une des forces de l'économie de la région ${ }^{27}$.

\section{L'épigraphie}

La première source épigraphique est fournie par une inscription grecque datée du $\mathrm{III}^{\mathrm{e}}$ siècle avant notre ère, mise au jour sur le site de Locres (fig. 2). Il s'agit d'une tablette de bronze provenant des archives de l'Olympeion de la cité ${ }^{28}$. On y trouve la mention de quarante kadoi de poix, à trois statères le kados $^{29}$. Ce texte est essentiel car il donne le nom du conteneur, kados. Les sources indiquent que le kados, cadus en latin, est un récipient destiné au stockage du vin ${ }^{30}$ ou des fruits ${ }^{31}$. Quelques textes évoquent également la matière : céramique pour Martial ${ }^{32}$, bronze pour Virgile ${ }^{33}$. Ces indications sont insuffisantes pour déterminer la forme du récipient, mais l'existence même d'un nom particulier montre que les Anciens ont éprouvé le besoin de le distinguer de l'amphore. Quant à sa contenance, on l'établit autour des 39 litres $^{34}$. L'impulsion des recherches sur les amphores ayant transporté la poix a été donnée par la découverte de timbres mentionnant la poix

26. Cassiodore, Variae, XII, 12.

27. R. Ragosta, Organizzazione e distribuzione commerciale del ferro, dell'acciaio e della pece in Calabria al tramonto del '600, dans Studi in memoria di Federigo Melis, IV, Naples, 1978, p. 367-394.

28. F. Costabile (dir.), Polis ed olimpieion a Locri Epizefiri. Costituzione economia e finanze di una città della Magna Grecia. Edition altera e traduzione delle tabelle locresi, Soveria Mannelli, 1992.

29. Il s'agit de la tablette n. 15. Voir F. Costabile, op. cit., p. 259.

30. Hérodote, Histoires, III, 20; Lucilius, Satires, XVIII, 556; Virgile, Enéide, I, 195.

31. Pline l'Ancien, Histoire naturelle, XIII, 48 et XV, 42.

32. Martial, Epigrammes, I, 55, 10.

33. Virgile, Enéide, VI, 228, éd. J. Perret, Paris, 1982 (Collection des Universités de France).

34. D'après E. Saglio (Ch. Daremberg et E. Saglio, Dictionnaire 


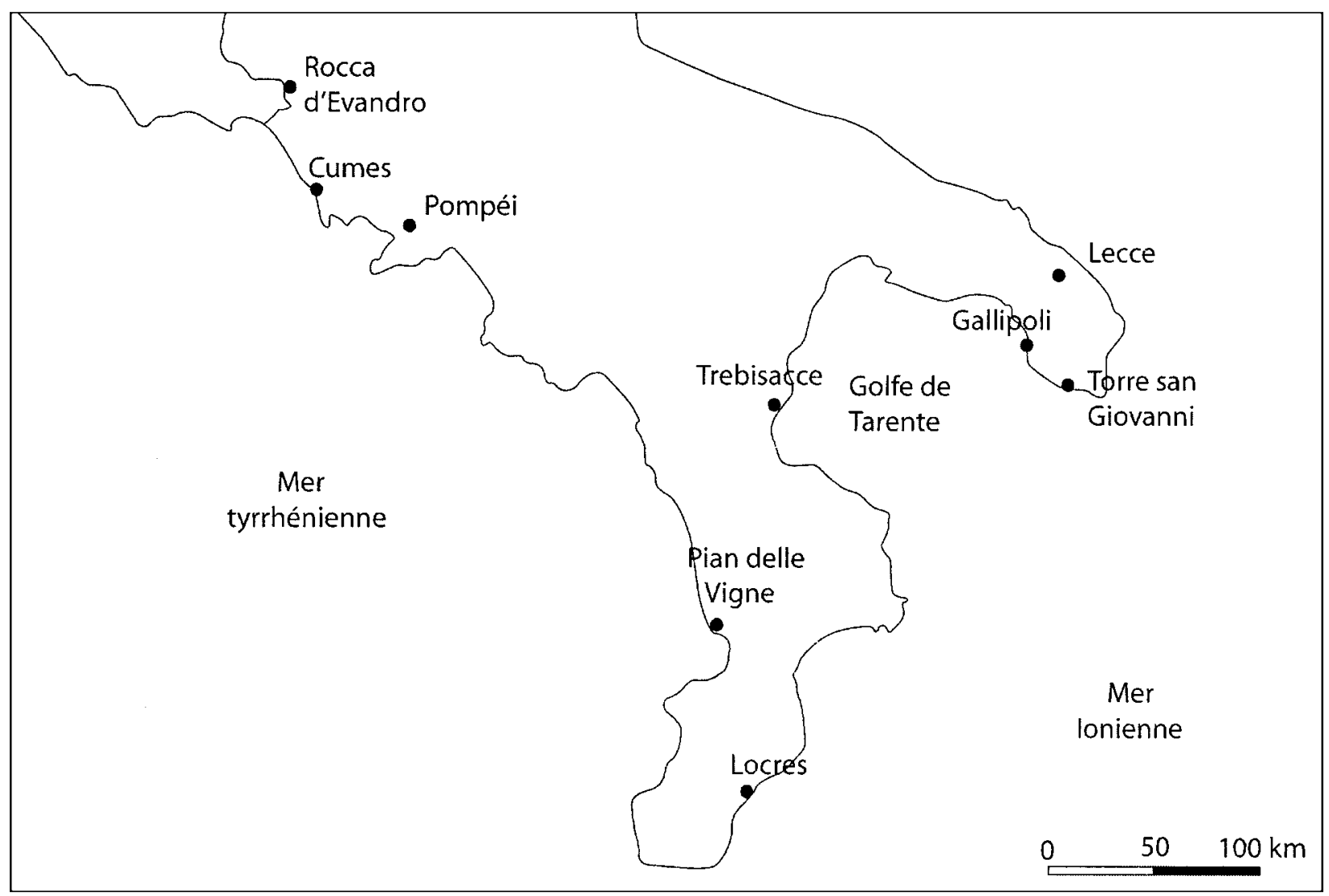

Fig. 2 - Carte du sud de l'Italie avec les sites mentionnés (dessin L. C.).

du Bruttium. Des quatre actuellement répertoriés, trois ont été mis au jour en Calabre, le dernier à Pompéi.

Deux timbres ont été découverts sur le site de "Pian delle Vigne», près de la ville de Falerna en Calabre (fig. 2). Le site correspond à une exploitation agricole occupée de la fin de la période hellénistique aux ${ }^{\text {er }}$ et $\mathrm{II}^{\mathrm{e}}$ siècles de notre ère. Les timbres proviennent de ramassages de surface ${ }^{35}$. Le premier exemplaire est imprimé sur le bord d'un conteneur. Le fragment qui mesure $7,6 \mathrm{~cm}$

des Antiquités grecques et romaines d'après les textes et les monuments, tome premier, deuxième partie, Paris, 1877, p. 778), un cadus équivaut au métrète attique soit trois urnes. F. Gaffiot (Dicitonnaire latin français, Paris, 1934, p. 237) propose les mêmes capacités soit, trois urnes ou 12 congii ou 72 sextarii. Or, nous savons qu'un métrète vaut 39,3 litres, et que le conge vaut 1/12 de métrète (N. Parise, trad. J. Andreau, Métrologie, Grèce et Rome, dans J. Leclant (dir.), Dictionnaire de l'Antiquité, Paris, 2005, p. 1411-1413). Le cadus vaut donc 39, 3 litres.

35. R. Spadea, L'area di Piano della tirena e di S. Eufemia Vetere, dans G. Maddoli (dir.), Temesa e il suo territorio, Atti del colloquio di Perugia e Trevi, 30-31 maggio 1981, Taranto, 1982, de hauteur et $6,7 \mathrm{~cm}$ de long, porte l'inscription : [.]IX BRVT (fig. 3a). L'interprétation est la même que pour les timbres suivants: [p]ix brut(tia). Le deuxième timbre se trouve sur un fragment de panse qui mesure $9,6 \mathrm{~cm}$ de haut pour $9,3 \mathrm{~cm}$ de longueur. On y lit $B R V T$ que l'on interprète comme (Pix) Brut(tia) ${ }^{36}$.

Le troisième timbre découvert en Calabre provient des fouilles d'un édifice de Trebisacce interprété comme un dépôt d'amphores (fig. 2) ${ }^{37}$. Il appartient à un contexte daté de la fin du ${ }^{\text {er }}$ siècle

p. 79-89, tav. 18-19. Voir plus particulièrement les pages 84-85.

36. F. Costabile, Altre attestazioni epigrafiche della pece bruzia, dans F. Costabile, op. cit., p. 173. À noter que dans l'édition de R. Spadea, le deuxième timbre est publié avec l'inscription $B R V T$. Dans l'édition de F. Costabile, le timbre est publié comme étant : PIX. BRVT.

37. A. Battista Sangineto publie à nouveau ce fragment en 2001 (A. Battista Sangineto, Trasformazioni o crisi nei Bruttii fra il II a.C. ed il VII d. C., dans E. Lo Cascio et A. Storchi Marino (dir.)., Modalità insiedative e strutture agrarie nell'Italia meridionale in età romana, Bari, 2001, p. 213, fig. 10) en l'identifiant, à tort, comme une amphore de type Dressel 21-22. Ces der- 


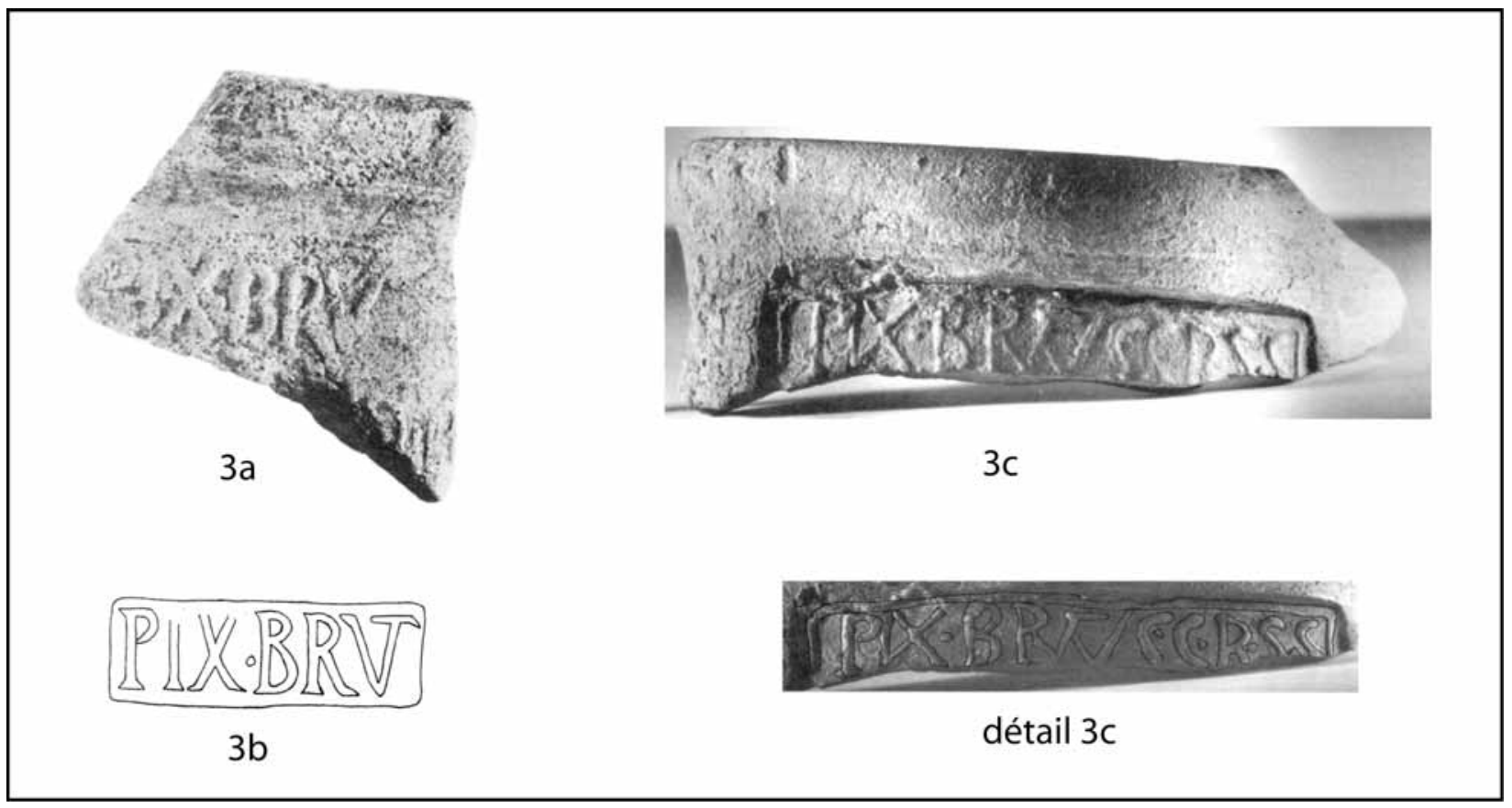

Fig. 3 - Les timbres mentionnant la poix du Bruttium provenant de Pian delle Vigne (3a, d'après F. Costabile 1992, p. 172, fig. 2), Pompéi (3b, d'après S. De Caro 1985, p. 24), et de Trebisacce (3c, d'après F. Costabile 1992, p. 172, fig. 3 et 4). Échelle 1:2.

de notre ère. L'inscription, plus développée, a donné lieu à de multiples interprétations (fig. 3c). Elle mesure $12,3 \mathrm{~cm}$ de long pour $1,8 \mathrm{~cm}$ de hauteur. Il s'agit d'un bord de conteneur ayant un diamètre d'ouverture d'environ $28 \mathrm{~cm}$. On peut lire : PIX.BRVTVC.C.R.S.S. La mention de la poix du Bruttium est bien acceptée. La fin de l'inscription a suscité sept restitutions ${ }^{38}$. Sur le site, quatre autres bords «d'amphores» sont signalés comme étant des conteneurs pour la poix ${ }^{39}$.

À l'extérieur de la Calabre, le seul timbre connu a été mis au jour à Pompéi (fig. 2) entre les années 1964 et 1970 par A. de Franciscis lors des fouilles de la maison de C. Iulius Polybius (Reg. IX, ins. 13, 1-3). Le fragment trouvé dans une pièce située autour de l'atrium a été publié en 1982 par S. de $\mathrm{Caro}^{40}$. Seul un tesson de panse de l'amphore est conservé. S. De Caro donne une description de la pâte : argile non locale, de couleur noi-

nières sont aujourd'hui bien identifiées comme des conteneurs ayant transporté des salaisons de poissons. Sur cet argument voir E. Botte, Le anfore Dressel 21-22 : anfore da pesce tirreniche nell'alto Impero, dans S. Pesavento-Mattioli et M.-B. Carre, Olio e pesce in epoca romana. Produzione e commercio nelle regioni dell'Alto Adriatico Atti del convegno internazionale di Padova (16 febb. 2007), à paraître. sette-marron foncé, avec de fines inclusions de mica. Le diamètre minimum du vase est de 20,6 $\mathrm{cm}$ et le diamètre maximum est de $32 \mathrm{~cm}$. Le timbre mesure $6,9 \mathrm{~cm}$ de long pour 2,4 de hauteur. De même que pour les deux timbres précédents, on y lit simplement PIX BRVT(tia), les lettres «u» et «t» étant ligaturées (fig. 3b). Ce fragment a été mis au jour dans les niveaux scellés par l'éruption de 79 de notre ère.

\section{L'APPORT DES FOUILLES SOUS-MARINES EN ITALIE DU SUD}

Comment identifier les conteneurs ayant servi au transport de la poix? Diverses découvertes effectuées au large les Pouilles attirent l'attention sur des conteneurs sans anse qui pourraient bien correspondre aux kadoi servant, entre autres, au transport de la poix. Huit kadoi baptisés «doliola»

38. F. Costabile, op. cit., p. 173. Nous ne nous attardons pas sur la lecture de ce timbre dans ce présent article.

39. A. Battista Sangineto, dans F. Costabile 1992 op. cit., p. 183 et tav. XXXVII, p. 184.

40. S. De Caro, Anfore per pece del Bruzio, dans Klearchos, XXVIII, 105-108, 1985, p. 21-32. 
par R. Auriemma nous intéressent plus particulièrement. Il s'agit d'un conteneur piriforme, à large ouverture, possédant un petit fond en bouton, et dépourvu d'anse. Ce dernier élément est particulièrement significatif car l'absence d'anse interdit de qualifier ces conteneurs d'amphores, le terme latin amphora, d'origine grecque, signifiant "grand vase à deux anses", "que l'on tient par les deux anses ${ }^{41}$. Nous proposons donc de les nommer kadoi.

Cinq de ces conteneurs ont été mis au jour lors de fouilles sous-marines au large de Torre San Giovanni (à quelques kilomètres au sud de Gallipoli) à plus de $37 \mathrm{~km}$ des côtes (fig. 2). Ils ne semblent pas faire partie d'une épave, mais R. Auriemma émet l'hypothèse qu'il s'agit d'une partie d'un chargement d'un navire citerne ${ }^{42}$, du genre des navires à dolia. Le diamètre d'ouverture est de 25 $\mathrm{cm}$ et ils sont datés entre la période augustéenne et le milieu du $\mathrm{I}^{\text {er }}$ siècle de notre ère. Bien que la publication ne fasse pas mention de poix à l'inté-

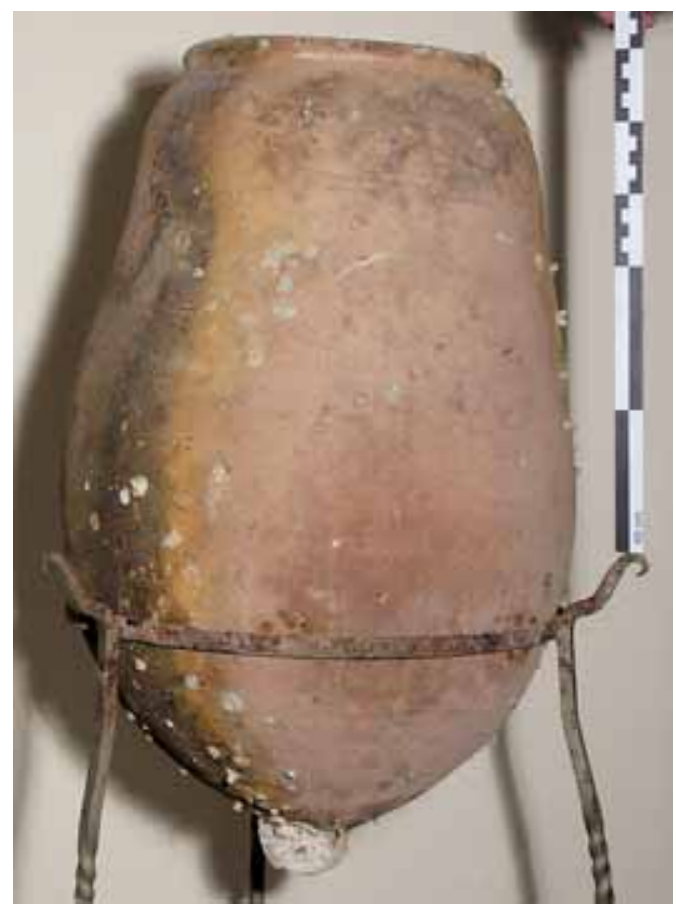

Fig. 4 - Un kados de Gallipoli (photo L. C.).

41. A. Bailly, Dictionnaire grec-français, Paris, 1985, s.v. amphora. 42. R. Auriemma, Salentum a salo, Forma maris antiqui, II, Lecce, 2004, p. 43-44.

43. R. Auriemma, Per la carta archeologica subacquea del Salento, dans Atti del convegno nazionale di archeologia subacquea, Anzio, 30-31 maggio e 1 giugno 1996, Bari, p. 232, fig. 12. rieur, une photo publiée indique clairement la présence de poix dans plus de la moitié du vase ${ }^{43}$.

Deux autres conteneurs, que nous avons eu l'occasion d'étudier attentivement, sont conservés au Musée archéologique de Gallipoli (fig. 2). Ils ont été découverts lors de fouilles sous-marines dans la zone du port de la ville. Le premier mesure $57 \mathrm{~cm}$ de hauteur, son diamètre d'ouverture est de $23,5 \mathrm{~cm}$, son diamètre maximum est de $35 \mathrm{~cm}$ (fig. 4 et 6a). Sa capacité maximale est de 36 litres. Le deuxième mesure $54 \mathrm{~cm}$ de hauteur, son diamètre d'ouverture est de $28 \mathrm{~cm}$, son diamètre maximum est de $33 \mathrm{~cm}$ (fig. 5 et $6 \mathrm{~b}$ ). Sa capacité maximale est de 33 litres $^{44}$. Leur contenance n'est donc pas très éloignée de celle établie pour le kados (soit 39 litres). Tous deux, dépourvus d'anse, sont remplis d'une poix très compacte ${ }^{45}$.

Un huitième conteneur est conservé au Musée provincial de Lecce $^{46}$. Il mesure $55,5 \mathrm{~cm}$ de hauteur, pour $19,5 \mathrm{~cm}$ de diamètre interne d'ouverture et $38,5 \mathrm{~cm}$ de diamètre maximum (fig. 6c). Il

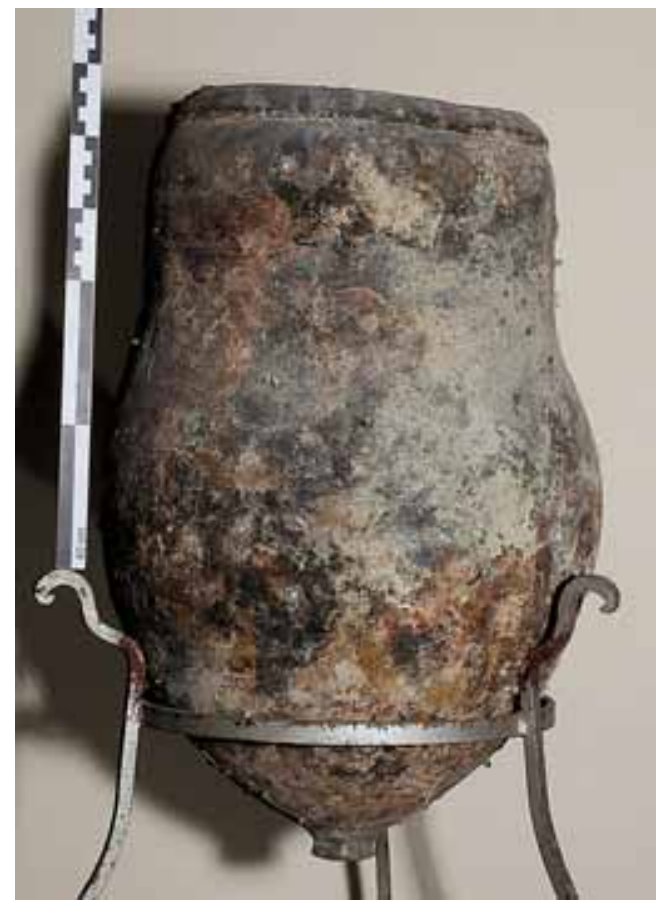

Fig. 5 - Un kados de Gallipoli (photo L. C.).

44. Les calculs de contenance ont été réalisés selon le modèle de Y. Rigoir. Voir Y. Rigoir, Méthode géométrique simple de calcul du volume des contenants céramiques, dans Documents d'Archéologie Méridionale, 4, 1981, p. 193-194.

45. L'un des deux est pratiquement rempli à moitié (figure 6a). 46. R. Auriemma, op. cit., 2004, p. 111, n. 321a et b. 


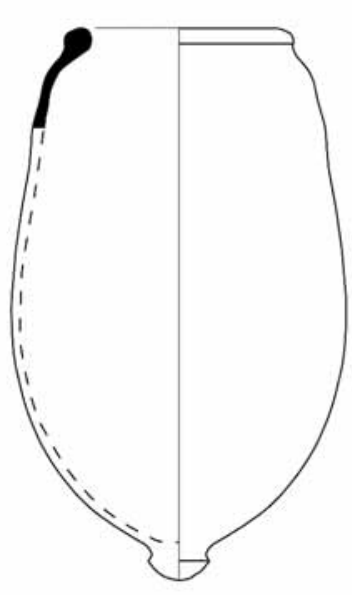

$6 a$

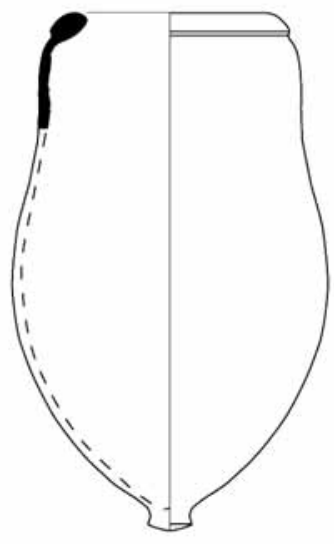

$6 b$

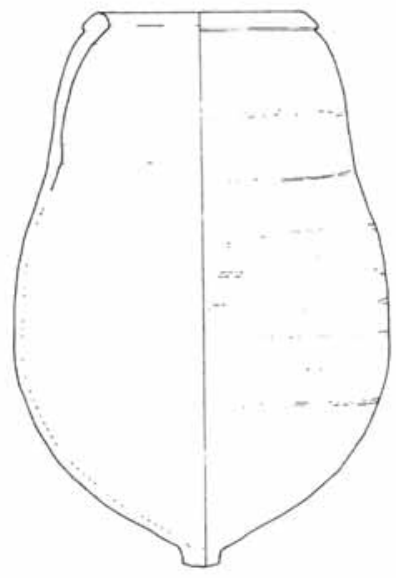

$6 c$

Fig. 6 - Les kadoi de Gallipoli (6a et 6b, dessins L. C.) et de Lecce (6c, d'après R. Auriemma 2004, p. 111, SRI 321b). Échelle 1:10.

possède des dimensions proches des exemplaires conservés à Gallipoli. Il n'est toutefois pas précisé, comme pour les exemplaires de Tarente, s'il contient de la poix.

Nous pouvons donc avancer l'hypothèse que ces kadoi ont servi au transport de la poix sous forme solide. Notre hypothèse est la suivante : la poix liquide ou semi-liquide est stockée dans ces vases où elle sèche et durcit. Les kadoi sont ensuite chargés dans les navires.

La poix contenue dans les kadoi voyageait sous forme solide ou au moins pâteuse. Si cela n'avait pas été le cas, elle se serait dispersée en mer au moment du naufrage. De telles observations ont été faites dans l'épave du Dramont $\mathrm{C}$, où une cargaison de poix liquide, certainement contenue dans des amphores, s'était répandue dans la coque du navire ${ }^{47}$. De plus, le diamètre d'ouverture des récipients et l'absence de bouchon plaident en faveur d'un contenu solide. Dans ce cas, comment la poix était extraite de ces conteneurs? Les kadoi étaient-ils mis à chauffer pour en extraire la poix? ou bien brisait-on les vases lors de l'utilisation?

Ces huit kadoi ayant été mis au jour en mer,

47. J.-P. Joncheray, L'épave Dramont C, Cahiers d'archéologie subacquatique, 12, Saint-Raphaël, 1994, p. 18.

48. E. Chiosi, G. Gasperetti, Rocca d'Evandro (Caserta)-Località Porto. Un quartiere artigianale romano sul fiume, dans Gl. Olcese (dir.), Ceramica romana e archeometria: lo stato degli nous supposons qu'ils appartenaient à des chargements de navires en partance du sud de l'Italie, notamment du massif du Salento, et destinés à des lieux de consommation que leur identification permet désormais de commencer à cerner.

Deux fragments ont été identifiés comme tels, par G. Gasperetti et E. Chiosi parmi le matériel mis au jour lors des fouilles d'un quartier artisanal le long du fleuve Garigliano, près de Rocca $\mathrm{d}^{\prime}$ Evandro $^{48}$. Le site se trouve à environ $30 \mathrm{~km}$ de Minturnes. Il s'agit de bords de conteneur. L'un mesure $17,6 \mathrm{~cm}$ de diamètre d'ouverture (fig. $7 \mathrm{a}$ ), le second 27,5 cm (fig. 7b). Aucune description de pâte n'est faite. Nous ne sommes donc pas en mesure de savoir s'il s'agit d'une production locale ou d'une importation de Calabre. Toutefois, les auteurs de l'article émettent l'hypothèse que ces conteneurs ont pu servir au transport de poix produite localement et non de la poix du Bruttium. Rappelons en effet que le site fouillé se trouve le long d'un axe fluvial, à quelques kilomètres de Minturnes où plusieurs inscriptions mentionnent la présence de picariorum sociorum ${ }^{49}$, et d'un architecte naval ${ }^{50}$. Or, nous savons bien aujourd'hui

studi, Florence, 1994, p. 293-299.

49. J. Johnson, Excavations at Minturnae, vol. II, Inscriptions, Part I, Republican magistri, Rome, 1933, n 7, 14, 16 et 19.

50. CIL X, 5371. 


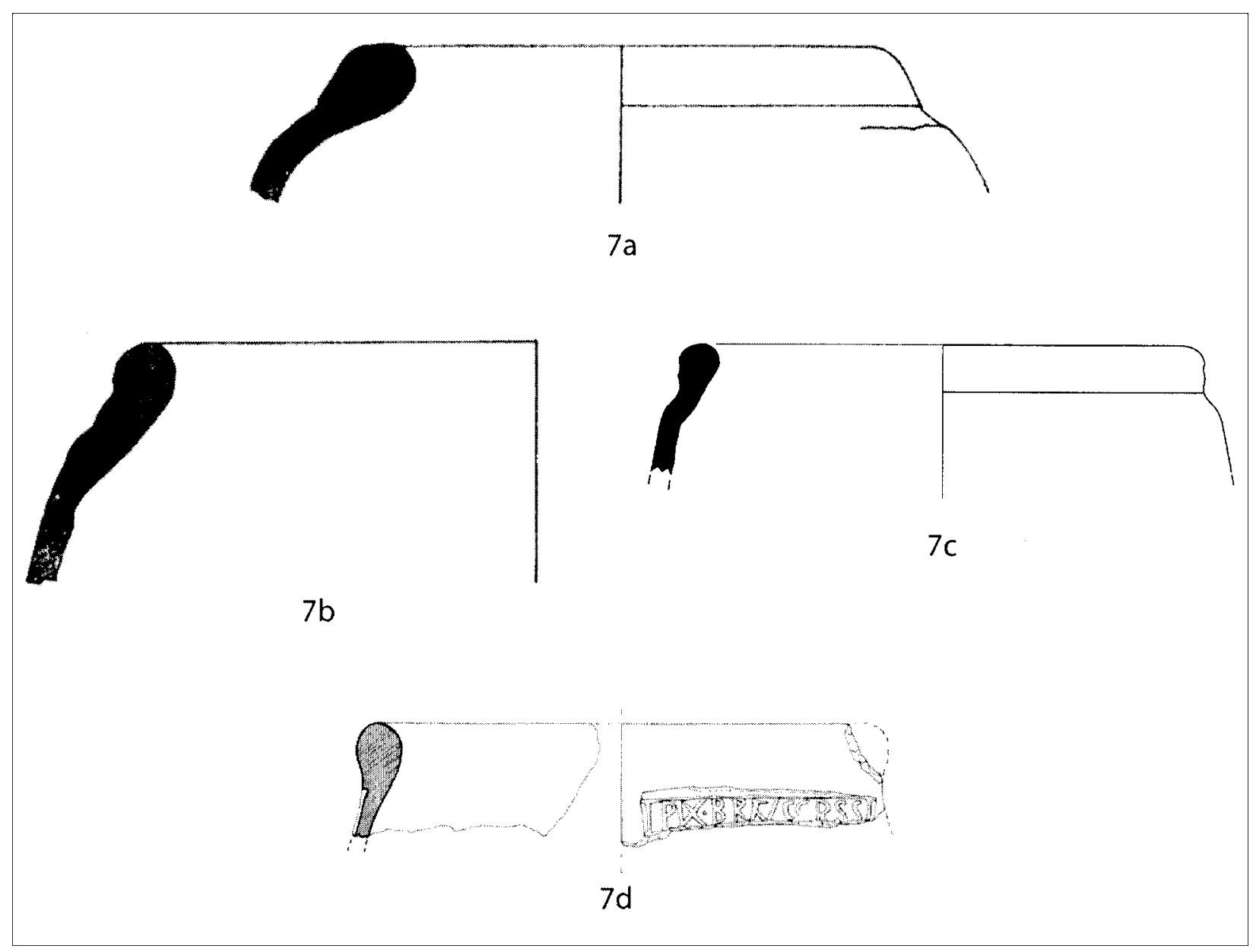

Fig. 7 - Les fragments de kadoi de Rocca d'Evandro (7a et 7b, d'après E. Chiosi et G. Gasperetti, 1994, p. 296, n. 11 et 12), de Cumes (7c, fouilles Centre Jean Bérard, dessin L. C.) et de Trebisacce (7d, d’après A. Battista Sangineto 1992, p. 184, tav. XXXVII, fig. 5, 47). Échelle 1:4.

que des dolia destinés à l'exportation ont été fabriqués dans la région ${ }^{51}$. La présence des picarii n'est donc pas dénuée d'importance, car la poix était utilisée pour imperméabiliser les dolia et les navires ${ }^{52}$. L'hypothèse avancée par G. Gasperetti et E. Chiosi prendrait évidemment tout son sens. Toutefois, un examen plus particulier de la pâte des exemplaires permettrait seul de s'en assurer. Le contexte de découverte n'est pas daté avec précision. Les éléments chronologiques du secteur fouillé s'étalent du II ${ }^{e}$ av. J.-C. au II ${ }^{e}$ siècle de notre ère.

Un autre fragment appartenant à cette forme a été mis au jour à Cumes dans les fouilles du Centre Jean Bérard. Il s'agit d'un fragment ayant un diamètre d'ouverture de $24,8 \mathrm{~cm}$ (fig. $7 \mathrm{c}$ ). La pâte est de couleur jaune-beige en surface et de couleur orangé-rouge au centre. Sa composition, avec de fines inclusions de mica, ne semble pas campanienne, mais plutôt calabraise. Ce fragment de kados a été mis au jour dans un niveau de remblai (us 700108) daté du Ier siècle de notre ère. Enfin, le fragment mis au jour à Trebisacce (fig. 7d) correspond tout à fait au profil des kadoi mentionnés et identifiés dans les Pouilles.

Le rapprochement entre les profils des soi-
51. M. Corsi-Sciallano et B. Liou, Les épaves de Tarraconaise à chargement d'amphores Dressel 2-4, dans, Archaeonautica 5, 1985, p. 171.

52. Les navires transportant les dolia ont peut-être même été fabriqués sur place. Voir M.-B. Carre et R. Roman, Hypothèse de restitution d'un navire à dolia : la construction d'une maquette, à paraître. 
disant "amphores» estampillées "poix du Bruttium" et les "doliola» mis au jour dans les Pouilles permet d'identifier le conteneur, kados, dans lequel était expédiée la poix du Bruttium et il fournit une clef pour rechercher les destinations de cette marchandise. Quant à la datation, elle repose surtout sur les timbres et les fragments découverts dans les fouilles terrestres puisque les individus complets trouvés en mer sont hors contexte. Les fragments de Rocca d'Evandro proviennent d'un contexte ayant une datation assez large, celui de Cumes est daté du Ir siècle de notre ère. Nous pouvons, en attente de nouveaux éléments, proposer une fourchette chronologique s'étendant du $\mathrm{I}^{\mathrm{er}}$ siècle avant Jésus Christ au $\mathrm{I}^{\mathrm{er}}$ siècle de notre ère ${ }^{53}$.

Laëtitia CAVAssa 